\title{
Europäisch eingestellt - Valenzforschung mit Parallelkorpora
}

\author{
Rolf Duffner/Alain Kamber/Anton Näf (Neuchâtel)
}

\begin{abstract}
The aim of this research is to demonstrate with a case study the significance of corpus linguistics within the field of verb valency and bilingual lexicography. Specifically, we will introduce a corpus-based process that determines context-sensitive translations of polysemous word forms. Three steps are considered here in detail. First, text evidences of the verb einstellen in the monolingual Deutsches Referenzkorpus (DeReKo) will be examined with a collocation analysis. With help of the analytical instrument COSMAS II, the collocation profiles will then be summarized into a typology (senses and subsenses, valency structures and typical collocations). In a further step, the determined senses can be attributed to the corresponding translations of the word form einstellen in other languages (English, French and Italian) by means of the multilingual parallel corpus Europarl (Open Source Parallel Corpus OPUS). Finally, the results will be compared to the codifications of commonly used bilingual dictionaries.
\end{abstract}

More data is better data.

(Church/Mercer)

\section{$1 \quad$ Einleitung}

Für Forschungen im Bereich der empirischen Sprachwissenschaft besteht seit einem knappen Jahrzehnt eine völlig neue Ausgangslage. Durch die Existenz und die zunehmend elaborierteren Abfragemöglichkeiten von großen digitalen Textdatenbanken eröffnen sich für eine adäquate Beschreibung von Grammatik und Lexikon von natürlichen Sprachen ganz neue Perspektiven. Dank der Möglichkeit eines schnellen Zugriffs auf riesige Mengen von Sprachdaten kann die Phase der Belegsammlung extrem verkürzt und dadurch Zeit für die Analyse und Interpretation der Rohdaten gewonnen werden. Korpuslinguistisch fundierte Forschungen sind nicht bloß von hohem theoretischem Interesse, sondern darüber hinaus von großem praktischen Nutzen, insbesondere für die Herstellung und die Bewertung von ein- und zweisprachigen Wörterbüchern. Dank der gegenwärtig verfügbaren Analysewerkzeuge kann man heute mit einem vertretbaren Aufwand überprüfen, ob die in den Wörterbüchern gemachten Angaben ein getreues Abbild von Vorkommen und Gebrauch der Lexeme in der Sprachwirklichkeit vermitteln oder nicht (für Fragen der Methodologie sei auf Kamber (2008) verwiesen).

Im vorliegenden Beitrag sollen Möglichkeiten des Einsatzes von korpuslinguistischen Verfahren in der Valenzforschung und in der zweisprachigen Lexikographie demonstriert werden. ${ }^{1}$ Bei der - für die Produktion von korrekten Äußerungen zentral wichtigen - Verbvalenz handelt es sich um ein sprachliches Phänomen, das an der Schnittstelle zwischen Grammatik und Lexikon anzusiedeln ist. Entsprechend kommt es nicht bloß in den Grammatiken zur Sprache,

\footnotetext{
1 Vgl. auch Duffner/Näf (2006), sowie Scherer (2006).
} 
sondern auch - oft auf mehr oder weniger implizite Weise - in den Wörterbüchern; explizit zum Thema gemacht wird es in den sogenannten Valenzwörterbüchern.

\section{Untersuchung}

Um die Phänomene der Verbvalenz und der Polysemie sowie deren Interdependenz in mehreren Sprachen miteinander vergleichen zu können, sind wir in der vorliegenden exploratorischen Studie in drei Schritten vorgegangen.

Anhand des exemplarisch herausgegriffenen polysemen Verbs einstellen soll hier ein korpusbasiertes Verfahren vorgestellt werden, mit dessen Hilfe den in einem Korpus vorkommenden Wortformen von einstellen die im jeweiligen Kontext gemeinte Unterbedeutung zugeordnet werden kann. Sodann werden die im mehrsprachigen Parallelkorpus Europarl ${ }^{2}$ auftretenden Übersetzungsäquivalente samt deren Kookkurrenzpartnern in den drei von uns ausgewählten Vergleichssprachen Englisch, Französisch und Italienisch ermittelt. Schließlich sollen die Angaben zum Verb einstellen in ausgewählten zweisprachigen Wörterbüchern mit den Korpusdaten konfrontiert werden.

Die beiden von uns herangezogenen Korpora sollen dabei nicht punktuell zur Verifizierung von Hypothesen konsultiert, sondern für die gewählte Fragestellung vollständig ausgewertet und analysiert werden.

\subsection{Erster Schritt: Wie wird das Verb einstellen im Deutschen gebraucht?}

Im einsprachigen Korpus DeReKo (Deutsches Referenzkorpus) $)^{3}$ werden alle Belegstellen für drei ausgewählte Verbformen des Lexems einstellen (nämlich einstellen, einzustellen und eingestellt $)^{4}$ gesammelt und durch Cosmas II einer automatischen Kookkurrenzanalyse unterzogen. ${ }^{5}$ Die drei von uns ausgewählten Verbformen machen im Gesamtkorpus ca. $75 \%$ aller Vorkommen für dieses Verb aus; von den verbleibenden Verbformen entfällt, wie eine Handauszählung bei einer Zufallsauswahl von 400 Belegen ergeben hat, etwa je die Hälfte auf univerbierte Verbformen (z. B. einstellte) bzw. auf solche in Klammerstellung (z. B. stellt ... ein).

Die mit Hilfe von Cosmas II gewonnenen Kookkurrenzprofile wurden zu einer - sowohl quantitative als auch qualitative Aspekte berücksichtigenden - Typologie (Unterbedeutungen, Valenzstrukturen, typische Kookkurrenzpartner) verdichtet; dabei beschränkten wir uns auf die 8 häufigsten Unterbedeutungen (vgl. Tab. 1).

2 Das Parallelkorpus Europarl 3 ist Teil des Open Source Parallel Corpus OPUS. Europarl besteht aus Sitzungsprotokollen des Europäischen Parlaments und enthält pro Sprache ca. 60 Mio. Textwörter. Für Informationen zu Europarl 3 und OPUS siehe Tiedemann.

3 Vgl. Institut für Deutsche Sprache (1999-2002).

4 Natürlich könnte man mit Cosmas II auch alle Flexionsformen von einstellen suchen; allerdings ist im Parallelkorpus Europarl 3 eine solche "Lemmasuche" nicht möglich, weshalb wir uns entschlossen haben, uns auf die drei genannten Verbformen zu konzentrieren.

5 Vgl. Institut für Deutsche Sprache. 


\begin{tabular}{|c|c|c|}
\hline Unterbedeutung & Bedeutungsangabe & Wichtige Kookkurrenzpartner \\
\hline 1.etw. einstellen & $\begin{array}{l}\text { mit etw. aufhören, etw. } \\
\text { nicht fortsetzen }\end{array}$ & $\begin{array}{l}\text { Verfahren (wegen Verjährung), Betrieb, Produktion } \\
\text { (vorübergehend), Ermittlungen (ergebnislos, mangels } \\
\text { Beweisen), Zahlungen, Geldbuße, Kampfhandlungen } \\
\text { (unverzüglich), Erscheinen, Arbeiten, Kämpfe, } \\
\text { Verkehr, Tätigkeit, Angriffe, Suche, Rauchen, } \\
\text { Bombardements, Feindseligkeiten [...] }\end{array}$ \\
\hline 2.jdn.einstellen & $\begin{array}{l}\text { in ein Arbeitsverhältnis } \\
\text { aufnehmen, anstellen }\end{array}$ & $\begin{array}{l}\text { Mitarbeiter, Lehrlinge, Personal, Arbeitskräfte, } \\
\text { Lehrer, Auszubildende, Behinderte (bevorzugt), } \\
\text { Arbeitslose, Ersatzkraft, Beschäftigte [...] / } \\
\text { zusätzliche, befristet }\end{array}$ \\
\hline $\begin{array}{l}\text { 3. sich auf jdn.letw. } \\
\text { einstellen }\end{array}$ & $\begin{array}{l}\text { sich auf etw. } \\
\text { vorbereiten }\end{array}$ & $\begin{array}{l}\text { darauf, Gegner, (neue) Situation, (veränderten) } \\
\text { Bedürfnisse }[\ldots] \text { / optimal, mental, bestens, taktisch } \\
\text { (hervorragend) }[\ldots]\end{array}$ \\
\hline 4. einstellen in etw. & $\begin{array}{l}\text { für etwas vorsehen, } \\
\text { budgetieren }\end{array}$ & $\begin{array}{l}\text { Haushalt, Etat, Nachtragshaushalt, } \\
\text { Haushaltsplan }[\ldots]\end{array}$ \\
\hline 5.etw. einstellen & regeln, justieren & $\begin{array}{l}\text { Visier, Kopfstützen, Skibindung, Außenspiegel [...] / } \\
\text { neu, richtig, manuell, stufenlos [...] }\end{array}$ \\
\hline 6.etw. einstellen & egalisieren & Rekord, Platzrekord [...] / von \\
\hline $\begin{array}{l}\text { 7. eingestellt sein } \\
\text { auf etw. }\end{array}$ & $\begin{array}{l}\text { eine bestimmte } \\
\text { Meinung / Gesinnung } \\
\text { haben }\end{array}$ & $\begin{array}{l}\text { positiv, kritisch, feindlich, skeptisch }[\ldots] / \\
\text { von Kopf bis Fuß auf Liebe, auf Sieg [...] / } \\
\text { gegenüber }\end{array}$ \\
\hline 8. sich einstellen & $\begin{array}{l}\text { eintreten, sich } \\
\text { einfinden }\end{array}$ & (erhoffte, gewünschte) Erfolge, Gratulanten [...] \\
\hline
\end{tabular}

Tabelle 1: Bedeutungsangaben und Kookkurrenzpartner zu den 8 häufigsten Unterbedeutungen des Verbs einstellen

Bei der Lektüre von Tabelle 1 ist Folgendes zu beachten:

Die Unterbedeutungen sind absteigend nach Frequenz und Typizität von 1 bis 8 durchnummeriert. Unterbedeutung 1 (mit etw. aufhören) ist also aufgrund der Korpusanalyse die häufigste und überdies die im Kookkurrenzprofil am stärksten repräsentierte Unterbedeutung. Die Kookkurrenzpartner ihrerseits sind ebenfalls absteigend nach Kohäsionsstärke aufgeführt und nach Satzgliedrolle (z. B. Akkusativobjekt, Adverbial) geordnet. Das Lexem einstellen in Unterbedeutung 1 hat also eine höhere Affinität zu Verfahren als zu Betrieb, Produktion usw. Mehrere Kookkurrenten treten kumulativ in Kookkurrenzclustern auf (z. B. Verfahren wegen Verjährung einstellen). Im Folgenden geben wir einige typische, zum Teil leicht verknappte Korpusbelege zu den einzelnen Unterbedeutungen.

1. Das Verfahren wurde wegen Geringfügigkeit eingestellt; Die Airline musste ihren Betrieb einstellen; Die Produktion musste vorübergehend eingestellt werden. Bei den Kookkurrenzpartnern handelt es sich überwiegend um Nomina in der Funktion von Akkusativobjekten. Wir haben uns hier auf Basisnomina wie Betrieb oder Verfahren beschränkt und die - allerdings oft typischen - Komposita wie Spielbetrieb, Liftbetrieb, Flugbetrieb, Strafverfahren, Ermittlungsverfahren usw. weggelassen. Adverbiale wie mangels Beweisen, wegen Geringfügigkeit oder vorübergehend sind ebenfalls einschlägige Kookkurrenzpartner zum Verb einstellen in Unterbedeutung 1. 
2. Das Unternehmen will zusätzliche Mitarbeiter einstellen; Im Herbst werden drei neue Lehrlinge eingestellt; Die Firma kann sich vorstellen, befristet ausländische Arbeitnehmer einzustellen. Bei Unterbedeutung 2 treten überwiegend Sammelbezeichnungen wie Mitarbeiter, Personal, Arbeitskräfte, Beschäftigte usw. auf. Erweiterungen wie zusätzliche oder befristet sind mit verschiedenen anderen Kookkurrenzpartnern kombinierbar.

3. Man muss sich schon im Training mental darauf einstellen; Die defensiven Grazer waren optimal auf den Gegner eingestellt; Auf diese neue Situation mussten wir uns erst einstellen.

4. 20'000 Mark waren dafür bereits im Haushaltsplan eingestellt worden; Die Gelder sind in den Etat einzustellen.

5. Kapitän Ivica Vastic hat das Visier wieder optimal eingestellt; Die meisten Kopfstützen sind nicht richtig eingestellt. Die Wendung das Visier optimal eingestellt haben wird in fast allen Fällen metaphorisch verwendet, beispielsweise für Fußballer, die besonders gut und häufig treffen.

6. Schumacher hat den legendären Rekord von Senna eingestellt; Platzrekord zum Auftakt eingestellt! Es handelt sich bei diesen Rekorden nur in wenigen Fällen um nichtsportliche Bestleistungen (z. B. Umsatzrekorde).

7. Er sei gegenüber dem Projekt positiv eingestellt; Ich bin von Kopf bis Fuß auf Liebe eingestellt. Das Marlene-Dietrich-Zitat kommt im Untersuchungskorpus vielfach abgewandelt vor: Die Leute sind nicht nur von Kopf bis Fuß auf Liebe, sondern auch auf Lügen, Sieg, Regen, Gesundheit und sogar auf Kürbis eingestellt.

8. Erste Erfolge haben sich bereits eingestellt; Zum hohen Geburtstag werden sich viele Gratulanten einstellen.

Das Dudenwörterbuch in 10 Bänden registriert insgesamt 10 zum Teil noch weiter unterteilte Unterbedeutungen, die teilweise als regional oder fachsprachlich markiert bezeichnet werden. Als erste Unterbedeutung wird in diesem Wörterbuch einstellen im Sinne von 'an einen bestimmten Platz stellen' genannt, eine Verwendungsweise, für die im Kookkurrenzprofil keine Kookkurrenten zu finden sind. Die in DeReKo mit Abstand am häufigsten belegte Unterbedeutung 'mit etw. aufhören' figuriert im Dudenwörterbuch an vierter Stelle.

\subsection{Zweiter Schritt (Teil 1): Welche Entsprechungen gibt es für das Verb einstellen in drei europäischen Sprachen?}

Die im ersten Schritt aus den sprachlichen Fakten ermittelte Typologie der Unterbedeutungen von einstellen wurde sodann im mehrsprachigen Parallelkorpus Europarl auf den deutschen Text und dessen Übersetzungsäquivalente in den drei Vergleichssprachen Französisch, Englisch und Italienisch projiziert. Durch dieses Verfahren lassen sich erstens Standardübersetzungen in den verschiedenen Vergleichssprachen gewinnen (Gleichungen wie einstellen : engager : to recrute : assumere), zweitens die (Quasi-)Synonyme innerhalb einer Sprache ermitteln (engager-embaucher-recruter) und drittens die jeweiligen Verben mit Hilfe der präferierten Kookkurrenzpartner gegeneinander abgrenzen (engager: personnes, personnel; recruter: personnel, jeunes). 
Valenzforschung mit Parallelkorpora

\begin{tabular}{|l|l|l|l|}
\hline $\begin{array}{l}\text { Zur Bekämpfung des } \\
\text { organisierten }\end{array}$ & $\begin{array}{l}\text { In the fight against } \\
\text { organised crime, more } \\
\text { Verbrechens wurden } \\
\text { recruited and some } \\
\text { mehr Spezialisten } \\
\text { eingestellt, und es } \\
\text { konnten mehrere } \\
\text { erfolgreiche Aktionen } \\
\text { gegen kriminelle } \\
\text { Netzwerke verbucht } \\
\text { criminal networks. } \\
\text { werden. }\end{array}$ & $\begin{array}{l}\text { Dans la lutte contre le } \\
\text { crime organisé, } \\
\text { davantage de } \\
\text { spécialistes ont été } \\
\text { recrutés, et certaines } \\
\text { actions menées contre } \\
\text { les réseaux criminels } \\
\text { ont été couronnées de } \\
\text { succès. }\end{array}$ & $\begin{array}{l}\text { Nella lotta contro la } \\
\text { criminalità organizzata } \\
\text { sono stati assunti più } \\
\text { specialisti e sono andate } \\
\text { a segno diverse azioni } \\
\text { contro reti criminali. }\end{array}$ \\
\hline $\begin{array}{l}\text { Im Gegenteil haben } \\
\text { neue Postbetreiber } \\
\text { eine große Anzahl an } \\
\text { Menschen eingestellt. }\end{array}$ & $\begin{array}{l}\text { On the contrary, new } \\
\text { postal operators have } \\
\text { taken on a large } \\
\text { number of people. }\end{array}$ & $\begin{array}{l}\text { Les nouveaux } \\
\text { opérateurs postaux ont } \\
\text { embauché un nombre } \\
\text { important de personnes. }\end{array}$ & $\begin{array}{l}\text { postali hanno assunto } \\
\text { parechie persone. }\end{array}$ \\
\hline
\end{tabular}

Tabelle 2: Ausschnitt aus Europarl 3. Die Belege sind bereits nach Unterbedeutung sortiert.

Von den 8 Unterbedeutungen aus DeReKo kommen in den deutschen Paralleltexten von Europarl - z. T. sicher auch korpusbedingt - nicht alle gleich häufig vor, wie aus Tabelle 3 hervorgeht:

\begin{tabular}{|c|c|c|c|c|c|}
\hline & Bedeutungsangabe & einstellen & eingestellt & einzustellen & Total \\
\hline 1. & mit etw. aufhören, etw. nicht fortsetzen & 150 & 390 & 267 & 807 \\
\hline 2. & $\begin{array}{l}\text { in ein Arbeitsverhältnis aufnehmen, } \\
\text { anstellen }\end{array}$ & 72 & 79 & 48 & 199 \\
\hline 3. & sich auf etw. vorbereiten & 162 & 42 & 111 & 315 \\
\hline 4. & für etw. vorsehen, budgetieren & 15 & 104 & 60 & 179 \\
\hline 5. & regeln / justieren & 13 & 0 & 10 & 23 \\
\hline 6. & (Sport) egalisieren & 0 & 0 & 0 & 0 \\
\hline 7. & $\begin{array}{l}\text { eine bestimmte Meinung / Gesinnung } \\
\text { haben }\end{array}$ & 0 & 67 & 0 & 67 \\
\hline \multirow[t]{2}{*}{8.} & eintreten, sich einfinden & 42 & 19 & 1 & 62 \\
\hline & Total & 454 & 701 & 497 & 1652 \\
\hline
\end{tabular}

Tabelle 3: Vorkommen von einstellen in Europarl 3, aufgegliedert nach Unterbedeutungen und Verbformen

Man kann hier zunächst einmal feststellen, dass die vier häufigsten Unterbedeutungen in Europarl auch in DeReKo die vier häufigsten Unterbedeutungen bilden. Unterbedeutung 6 (einen Rekord egalisieren) fehlt in Europarl dagegen völlig.

Zum weiteren Verfahren: Nachdem alle 1652 Belege für einstellen, eingestellt und einzustellen in Europarl "von Hand" einer der 7 belegten Unterbedeutungen zugeordnet worden sind, können 7 Teilkorpora zusammengestellt werden, in denen das Lexem einstellen ausschließlich in der betreffenden Unterbedeutung vorkommt. In diesen Teilkorpora können dann die in den Vergleichssprachen auftretenden Entsprechungen identifiziert und in eine Rangliste gebracht werden. In den Tabellen 4 bis 7 sind die Ergebnisse dieser Teilprozedur für die vier häufigsten Unterbedeutungen zusammengefasst. 


\begin{tabular}{|lr|}
\hline Englisch \\
\hline to stop & 192 \\
\hline to cease & 61 \\
\hline to suspend & 57 \\
\hline to end & 37 \\
to come to an end & 5 \\
\hline to halt & 24 \\
to call a halt & 9 \\
\hline to close & 30 \\
\hline to discontinue & 28 \\
\hline to withdraw & 25 \\
\hline & \\
\hline andere Verben & 106 \\
\hline Total Verben & 574 \\
\hline
\end{tabular}

\begin{tabular}{|lr|}
\hline Französisch \\
\hline cesser & 148 \\
\hline suspendre & 90 \\
\hline mettre un terme & 84 \\
arriver à son terme & 1 \\
\hline arrêter & 81 \\
\hline mettre fin & 47 \\
prendre fin & 14 \\
\hline supprimer & 49 \\
\hline interrompre & 35 \\
\hline abandonner & 30 \\
\hline \multicolumn{2}{|l}{} \\
\hline andere Verben & 73 \\
\hline Total Verben & 652 \\
\hline
\end{tabular}

\begin{tabular}{|lr|}
\hline Italienisch \\
\hline sospendere & 144 \\
\hline cessare & 122 \\
\hline porre fine & 79 \\
mettere fine & 13 \\
avere fine & 2 \\
\hline interrompere & 73 \\
\hline smettere & 37 \\
\hline eliminare & 31 \\
\hline bloccare & 29 \\
\hline abbandonare & 27 \\
\hline & \\
\hline andere Verben & 84 \\
\hline Total Verben & 641 \\
\hline
\end{tabular}

Tabelle 4: Übersetzungsäquivalente von Unterbedeutung 1 mit etw. aufhören, etw. nicht fortsetzen (807 Okkurrenzen)

\begin{tabular}{|lr|}
\hline Englisch \\
\hline to employ & 54 \\
\hline to recruit & 46 \\
\hline to take on & 23 \\
\hline to hire & 14 \\
\hline & 15 \\
\hline andere Verben \\
\hline Total Verben \\
\hline Diverses: \\
\hline
\end{tabular}

\begin{tabular}{|lr|}
\hline Französisch \\
\hline engager & 66 \\
\hline embaucher & 47 \\
\hline recruter & 31 \\
\hline nommer & 3 \\
\hline & 1 \\
\hline andere Verben & 148 \\
\hline Total Verben & 51 \\
\hline Diverses:
\end{tabular}

\begin{tabular}{|lr|}
\hline Italienisch \\
\hline assumere & 121 \\
\hline reclutare & 12 \\
\hline impiegare & 9 \\
\hline nominare & 5 \\
\hline & 3 \\
\hline andere Verben & 150 \\
\hline Total Verben & 49 \\
\hline Diverses:
\end{tabular}

Tabelle 5: Übersetzungsäquivalente von Unterbedeutung 2 in ein Arbeitsverhältnis aufnehmen (199 Okkurrenzen)

\begin{tabular}{|lr|}
\hline Englisch \\
\hline to adapt & 66 \\
\hline to prepare & 57 \\
\hline to adjust & 28 \\
\hline to anticipate & 16 \\
\hline to respond & 6 \\
\hline to expect & 5 \\
\hline to gear & 5 \\
\hline & \\
\hline andere Verben & 27 \\
\hline Total Verben & 210 \\
\hline Diverses & 105 \\
\hline
\end{tabular}

\begin{tabular}{|lr|}
\hline Französisch \\
\hline s'adapter & 102 \\
\hline se préparer & 54 \\
\hline anticiper & 16 \\
\hline faire face & 8 \\
\hline être prêt & 8 \\
\hline répondre & 8 \\
\hline s'attendre & 7 \\
\hline & \\
\hline andere Verben & 25 \\
\hline Total Verben & 228 \\
\hline Diverses & 87 \\
\hline
\end{tabular}

\begin{tabular}{|lr|}
\hline Italienisch \\
\hline adeguarsi & 62 \\
\hline prepararsi & 44 \\
\hline adattarsi & 39 \\
\hline anticipare & 12 \\
\hline far fronte & 8 \\
\hline fronteggiare & 1 \\
\hline essere pronto & 9 \\
\hline & \\
\hline andere Verben & 31 \\
\hline Total Verben & 206 \\
\hline Diverses & 109 \\
\hline
\end{tabular}

Tabelle 6: Übersetzungsäquivalente von Unterbedeutung 3 sich auf etw. vorbereiten (315 Okkurrenzen) 
Valenzforschung mit Parallelkorpora

\begin{tabular}{|lr|}
\hline Englisch \\
\hline to put & 25 \\
\hline to include & 21 \\
\hline to place & 13 \\
\hline to allocate & 12 \\
\hline to enter & 12 \\
\hline to earmark & 10 \\
\hline & \\
\hline andere Verben & 33 \\
\hline Total Verben & 126 \\
\hline Diverses & 53 \\
\hline
\end{tabular}

\begin{tabular}{|lr|}
\hline Französisch \\
\hline inscrire & 38 \\
\hline mettre & 23 \\
\hline placer & 17 \\
\hline prévoir & 10 \\
\hline affecter & 5 \\
\hline consacrer & 5 \\
\hline & \\
\hline andere Verben & 35 \\
\hline Total Verben & 133 \\
\hline Diverses & 46 \\
\hline
\end{tabular}

\begin{tabular}{|lr|}
\hline Italienisch \\
\hline iscrivere & 80 \\
\hline inserire & 15 \\
\hline destinare & 10 \\
\hline mettere & 7 \\
\hline prevedere & 6 \\
\hline stanziare & 6 \\
\hline & \\
\hline andere Verben & 29 \\
\hline Total Verben & 153 \\
\hline Diverses & 26 \\
\hline
\end{tabular}

Tabelle 7: Übersetzungsäquivalente von Unterbedeutung 4 für etw. vorsehen, budgetieren (179 Okkurrenzen)

Im Folgenden sollen exemplarisch einige aus den Tabellen 4 bis 7 ableitbare Erkenntnisse zur Sprache kommen. Nicht eingegangen wird auf die unter Diverses subsumierten Belege, in denen das Signifikat von einstellen nicht durch ein Verb, sondern auf andere Weise (insbesondere durch eine Nominalkonstruktion) ausgedrückt wird. Bei Unterbedeutung 3 (sich auf etw. vorbereiten) sind solche nichtverbalen Entsprechungen relativ zahlreich. Unterbedeutung 4 (Geld für etw. vorsehen) stellt insofern einen Spezialfall dar, als der damit bezeichnete Vorgang nicht durch das Verb einstellen allein, sondern durch das Syntagma in den Haushalt, in den Etat usw. einstellen versprachlicht wird (in der Schweiz würde man eher von budgetieren, franz. budgét(is)er sprechen); man kann hier von einer Mehrworteinheit sprechen, die in den Vergleichssprachen ebenfalls meist durch eine solche wiedergegeben ist.

Schon bei einer bloß summarischen Betrachtung der obigen Tabellen fällt auf, dass bei einzelnen Übersetzungsäquivalenten ein einzelnes Verb klar dominiert und damit gewissermaßen als Standardübersetzung gelten kann, etwa ${ }^{1}$ einstellen : to stop ${ }^{3}$ einstellen : s'adapter oder ${ }^{2}$ einstellen : assumere. So findet sich z. B. im englischen Parallelkorpus in fast jedem vierten Beleg to stop als Äquivalent von ${ }^{1}$ einstellen, während die Verben to cease, to suspend und to end deutlich seltener gebraucht werden. In anderen Fällen gibt es aber keinen so klaren Tabellenleader. So sind etwa bei ${ }^{2}$ einstellen die englischen Entsprechungen to employ und to recruit praktisch gleich häufig, und Entsprechendes gilt im Französischen für ${ }^{2}$ einstellen : engager, embaucher und im Italienischen für ${ }^{l}$ einstellen : sospendere, cessare.

Sind nun diese Entsprechungen innerhalb einer Unterbedeutung in allen Fällen gegeneinander austauschbar und somit gleichbedeutend? Oder hängt die Wahl des entsprechenden Verbs vom sprachlichen Kontext in den Vergleichssprachen ab? Um auf solche Fragen Antworten zu finden, wurden die nach Unterbedeutung geordneten Teilkorpora einer Kookkurrenzanalyse unterzogen. Auf diesem Weg können jeder Entsprechung in den Vergleichssprachen ein oder mehrere Kookkurrenzpartner zugeordnet werden. Im nächsten Schritt werden wir uns also nicht mehr nur mit Charakterisierungen rein quantitativer Natur begnügen, sondern die konkreten Kotexte auch qualitativ analysieren.

\subsection{Zweiter Schritt (Teil 2): Welches sind die typischen Kookkurrenzpartner der Übersetzungsäquivalente von einstellen?}

In den nachfolgenden drei Tabellen 8 bis 10 werden Kookkurrenzpartner zu den frequentesten Entsprechungen innerhalb der Unterbedeutungen 1, 2 und 3 aufgeführt. Diese Kookkurrenz- 
partner wurden in den Teilkorpora mit Hilfe des Programms Wordsmith ${ }^{6}$ ermittelt und sind hier absteigend nach Kohäsionswert angeordnet.

\section{Englisch}

to stop (192 Belege)

paying, giving, funding; aid; executions, activities

(1) [...] obviously, what the Commission cannot do when faced with this sort of difficulty is decide to stop paying, because that would be a bureaucratic solution.

(2) Some of you wish to stop aid immediately, and in particular operating aid, and a number of governments defend this point of view even today.

(3) Equally, European Union leaders are right to call on Israel to withdraw its military forces, stop extrajudicial executions, lift closures and restrictions on the Palestinian people, free settlements and end operations directed against Palestinian infrastructure.

to cease (61 Belege)

hostilities

(4) It strongly urges the two countries not to resort to violence, to cease all hostilities and to resume negotiations.

to suspend (57 Belege)

cooperation

(5) Iran's recent decisions to resume the uranium enrichment activities and to suspend all voluntary cooperation with the Agency are particularly worrying [...].

Französisch $^{7}$

cesser (148 Belege)

activités, violence, hostilités

(6) Depuis deux ans, le Hamas a déclaré et respecté un cessez-le-feu, il a cessé ses activités.

(7) Tous les groupes armés illégaux doivent cesser les hostilités.

suspendre (90 Belege)

activité, aide

(8) La rupture des négociations n'a pas eu un impact négatif comparable à celui observé à la fin de l'année 1996, lorsque toutes les activités de pêche ont été suspendues.

(9) Je voudrais attirer une nouvelle fois votre attention sur le fait que nous n'avons pas suspendu l'aide aux Palestiniens, comme d'aucuns l'ont prétendu.

arrêter (81 Belege)

production

(10) Il faut arrêter toute production de ces armes et détruire tous les stocks.

Italienisch

sospendere (144 Belege)

attività, aiuti

(11) Se per ragioni militari questo sistema è scollegato, le imprese europee sono costrette a sospendere molte delle loro attività.

(12) L'Unione europea ha sospeso gli aiuti al Burundi nel gennaio 1997 per poi riattivarli a seguito dei negoziati di pace.

cessare (122 Belege)

attività, produzione

(13) Nel corso dell ultimo anno e mezzo, 15 imprese del settore dell elettronica e imprese correlate hanno cessato le attività di produzione in Europa.

(14) Inoltre, in Europa, alcuni paesi cesseranno la produzione mentre altri la proseguiranno con difficoltà, sia per i produttori sia per le imprese e i lavoratori.

6 Vgl. www.lexically.net/wordsmith/.

7 Die Analyse hat keine Kookkurrenzpartner für das frequente Syntagma mettre un terme zutage gefördert. 
porre fine (79 Belege); mettere fine (13 Belege); avere fine (2 Belege)

violenza

(15) Invitiamo tutte le parti a porre fine alla violenza e a rilasciare gli ostaggi, sia europei che non europei, perché la dignità umana è un diritto per tutti.

interrompere (73 Belege)

attività

(16) Non si dice quindi che le attività sono già state interrotte.

Tab. 8: Unterbedeutung 1 (mit etw. aufhören) mit Kookkurrenzpartnern

Englisch

to employ (54 Belege)

people

(17) I started work there in 1967 when it employed 25'000 people, and now I walked into an empty factory.

to recruit (46 Belege)

staff

(18) According to the report, the Commission would need to recruit staff as a result and would be given the corresponding funds.

\section{Französisch}

engager (66 Belege)

personnes, personnel

(19) Les employeurs évitent ainsi d'engager davantage de personnes pour répartir les tâches entre elles.

(20) La législation communautaire devra être traduite dans les langues des nouveaux États membres et il faudra engager du personnel provenant de ces derniers, sans dépasser le cadre budgétaire convenu.

embaucher (47 Belege)

personnel

(21) Grâce à ces mesures, nous disposons d'un marché du travail flexible, au sein duquel il est facile de licencier, mais plus facile encore d'embaucher du personnel.

recruter (31 Belege)

personnel, jeunes

(22) Il faudrait selon lui que la Commission recrute du personnel en conséquence, et se voie dotée des crédits correspondants.

(23) Pour les grades inférieurs - dans la carrière A, j'entends - on a recruté des jeunes sans expérience professionnelle ou avec peu d'expérience.

Italienisch

assumere (121 Belege)

personale, persone, giovani, donne

(24) Spesso è anche la famigerata giungla di regole, moduli e sportelli che scoraggia il piccolo imprenditore dall'assumere nuovo personale.

(25) Esso ci ha dotato di un mercato del lavoro flessibile in cui è facile licenziare, e ancora più facile assumere persone.

reclutare (12 Belege)

personale

(26) Indubbiamente uno o due degli emendamenti sono assurdi; in uno, per esempio, si afferma la necessità di reclutare personale che sia capace di occuparsi di petizioni redatte in gallese.

Tab. 9: Unterbedeutung 2 (in ein Arbeitsverhältnis aufnehmen) mit Kookkurrenzpartnern 
Englisch

to adapt (66 Belege)

situation, market, change // new // time to, must

(27) I believe that the text, in the terms in which it provides for inclusion, is intended to achieve both the elimination of unfair competition and the granting of a prudent time limit for the self-employed to adapt to the new situation and to ensure their protection.

(28) We must adapt to these circumstances.

(29) We are going to see the use of future profits phased out over an eight- or nine-year period, which will give the industry time to adapt to the new conditions.

to prepare (57 Belege)

themselves // need, must, should

(30) But what we need to prepare ourselves for, what my British colleagues need to prepare themselves for, is the need to find a sensible way to have those parts of the rebate phased out which are not 'agricultural', namely regional and structural policy and thus solidarity within the Community, and future enlargement to the East.

(31) They finally want to be in charge of their region, their country, and we should be prepared for this.

to adjust (28 Belege)

new // time to

(32) In retrospect, therefore, we can see that we might have been wiser to have given this important project more time and waited not only until the ten new Member States had settled in but also until all twenty-five members had had time to adjust to the new realities of the European Union.

Französisch ${ }^{8}$

s'adapter (102 Belege)

situation, marché, changement // nouvelle // devons

(33) Toutefois, il en résulte aussi une situation nouvelle à laquelle le monde économique et les législateurs nationaux doivent s'adapter.

(34) Bien que la Commission soit fermement convaincue des bienfaits de la fin du système des conférences, elle est également convaincue de la nécessité de donner à l'industrie suffisamment de temps pour s'adapter à un marché placé sous le signe de la concurrence.

se préparer (54 Belege)

devons

(35) Nous devons nous préparer l'an prochain à une augmentation de la taille du Parlement européen en 2004 de plus d'une centaine de membres, qui apporteront avec eux dix nouvelles langues.

Italienisch

adeguarsi (62 Belege)

mercato // nuovo // dovranno

(36) L'agricoltura non è come un negozio di biciclette, che può con facilità adeguarsi alle esigenze del mercato.

(37) Condivido l'opinione che vi sia l'esigenza di posporre la data di entrata in vigore, in modo da permettere alle aziende di adeguarsi alle nuove regole.

prepararsi (44 Belege)

dobbiamo

(38) A quale scadenziario dobbiamo prepararci?

(39) Vogliono finalmente occuparsi della propria regione e del proprio paese, e dobbiamo prepararci a questa evenienza.

adattarsi (39 Belege)

situazione, cambiamenti // nuove

(40) Credo che il testo, stabilendo l'inclusione nei termini previsti, abbia lo scopo di eliminare la concorrenza sleale e, al tempo stesso, concedere un adeguato periodo all'autonomo affinché possa adattarsi alla nuova situazione e ne venga assicurata la protezione.

Tab. 10: Unterbedeutung 3 (sich auf etw. vorbereiten) mit Kookkurrenzpartnern

$\mathrm{Zu}$ diesen Angaben und Beispielen folgende Lesehinweise: Bei den - kursiv gesetzten -Kookkurrenzpartnern handelt es sich meist um Akkusativobjekte, teilweise aber auch um solche

8 Die Analyse hat keine Kookkurrenzpartner für anticiper zutage gefördert. 
mit anderen Funktionen, z. B. Modalverben bei Unterbedeutung 3 (must, should, devons, dobbiamo), welche die Notwendigkeit zur Anpassung zum Ausdruck bringen.

Die obigen Resultate der Kookkurrenzanalyse (und die zitierten Beispiele) ermöglichen nun Aussagen über typische Kookkurrenten der einzelnen Übersetzungsentsprechungen. So zeigt etwa das Verb to employ eine Affinität zu people, to recruit dagegen eine solche zu staff. Im Französischen ist offenbar die Kollektivbezeichnung personnel mit allen Verben (engager, embaucher, recruter) kompatibel, während personnes und jeunes diesbezüglich Präferenzen aufweisen.

Aber auch interlinguale Beobachtungen können von Interesse sein. Bei Unterbedeutung 3 (sich auf etw. vorbereiten) sind etwa zwischen Englisch, Französisch und Italienisch Übereinstimmungen zu konstatieren, und zwar nicht bloß bezüglich des Etymons der Verben, sondern darüber hinaus auch der Kookkurrenzpartner, z. B. bei den Gleichungen to adapt : s'adapter oder to prepare : se préparer : prepararsi.

\subsection{Dritter Schritt: Was für Angaben zum Verb einstellen finden sich in zweisprachi- gen Wörterbüchern?}

Die anhand des einsprachigen und des zweisprachigen Korpus ermittelten Ergebnisse sollen nun mit den Kodifizierungen in ausgewählten zweisprachigen Wörterbüchern verglichen werden. Es geht um die Beantwortung der Frage: Sind die darin enthaltenen Angaben richtig, zuverlässig und angemessen?

Im Folgenden werden zunächst die Langenscheidt e-Handwörterbücher Deutsch - Englisch, Deutsch - Französisch und Deutsch - Italienisch untersucht, anschliessend das Larousse Wörterbuch Deutsch - Französisch von Grappin et al. (1999).

Die drei Langenscheidt-Wörterbücher setzen unterschiedlich viele Unterbedeutungen an (Englisch: 9, Französisch: 7 und Italienisch 18). In allen drei Nachschlagewerken wird übrigens ein transitiver von einem reflexiven Gebrauch unterschieden (im unten reproduzierten Textausschnitt jeweils durch einen Abstand abgetrennt). Der folgende Vergleich beschränkt sich wiederum auf die vier im Parallelkorpus untersuchten Unterbedeutungen, die hier aber in der Originalnummerierung der Wörterbücher zitiert werden. An erster Stelle steht übrigens in allen drei von uns herangezogenen Wörterbüchern (wohl nach dem Vorbild des DudenWörterbuchs) die - in unseren beiden Korpora nicht belegte - Unterbedeutung hineinstellen .

2. (Arbeitskräfte etc.) take on, hire

3. (beenden) stop; (Produktion) auch cease, discontinue; (Feindseligkeiten, Kampfhandlungen) end, cease; (Buslinie, Zugverkehr) discontinue, close down; (Streik, Suche), call off; etwas vorïbergehend einstellen suspend s.th. temporarily; die Arbeit einstellen Person: stop work; (streiken) down tools; Fabrik: cease production; das Feuer einstellen MIL cease fire, stop shooting (oder firing); das Verfahren einstellen JUR suspend proceedings, drop the case; bitte stellen Sie jetzt das Rauchen ein please stop smoking now; die Zeitung hat ihr Erscheinen eingestellt the newspaper has ceased publication

2. sich einstellen auf $(+A k k$.) (sich anpassen an) adapt (oder adjust) (o.s. oder itself) to; (sich vorbereiten auf) prepare (o.s.) for, get ready for, gear (o.s.) up for fam.; (rechnen mit) be prepared for; (Aufmerksamkeit darauf richten) focus one's attention on; (Lebenstil) adjust one's lifestyle (oder way of thinking) to; sich auf einen Gegner einstellen prepare to face an opponent; $\boldsymbol{d u}$ musst dich darauf einstellen (daran gewöhnen) you'll have to get used to it (oder learn to accept it); eingestellt $I I$, einrichten $I I 3$

Langenscheidt Deutsch - Englisch 
Von den vier oben untersuchten Unterbedeutungen führt Langenscheidts Handwörterbuch Deutsch - Englisch nur deren drei auf: Die vierthäufigste (für etw. vorsehen, budgetieren) kommt darin nicht vor.

Für Unterbedeutung 1 (mit etw. aufhören) werden neben dem prototypischen to stop auch die Übersetzungen to cease, to discontinue und to end mit möglichen Kollokationspartnern aufgeführt. Man fragt sich allerdings, wieso diese in der Ausgangssprache geboten werden (beispielsweise to cease mit Feindseligkeiten) und nicht direkt auf Englisch.

Unterbedeutung 2 (in ein Arbeitsverhältnis aufnehmen) wird mit den beiden Übersetzungsvorschlägen to take on und to hire nur knapp behandelt, ohne Angabe von Kookkurrenzpartnern. Die zwei frequentesten Entsprechungen in Europarl (to employ und to recruit) fehlen.

Bei Unterbedeutung 3 (sich auf etwas vorbereiten) kommen gleich fünf in Europarl auftretende Entsprechungen vor, unter ihnen die drei Spitzenreiter to adapt, to adjust und to prepare.

3. Arbeitskräfte engager; embaucher

4. (beenden, aufhören) cesser; arrêter; supprimer; Zahlungen etc suspendre; JUR Verfahren arrêter

2. sich auf etwas (acc) einstellen s'adapter à qc; se préparer à qc; s'attendre à qc; auf etwas (acc) eingestellt sein être prêt à qc; être organisé en vue de, pour qc; sich auf jemanden einstellen s'adapter à qn; se régler sur qn; gegen jemanden eingestellt sein être mal disposé envers qn; sozialistisch eingestellt sein avoir des idées socialistes

Langenscheidt Deutsch - Französisch

Auch in Langenscheidts Handwörterbuch Deutsch - Französisch fehlt Unterbedeutung 4 (für etw. vorsehen, budgetieren).

Für Unterbedeutung 1 werden vier in Europarl frequente Verben genannt (cesser, arrêter, supprimer und suspendre); die in diesem Korpus prominent vertretenen Funktionsverbgefüge mettre un terme / arriver à son terme und mettre fin / prendre fin kommen dagegen nicht vor. Die Verben cesser, arrêter und supprimer werden zunächst ganz ohne Verwendungskontexte aufgelistet, was das Risiko in sich birgt, dass sie vom Wörterbuchbenutzer als Synonyme verstanden werden könnten. Zum Übersetzungsvorschlag suspendre wird Zahlungen als Kookkurrent aufgeführt, während in Europarl aide und activité als Partner belegt sind. Der juristische Gebrauch von arrêter kann durch die Korpusanalyse nicht bestätigt werden ${ }^{9}$; in Europarl ist das Substantiv production der wichtigste Kookkurrent von arrêter.

Für Unterbedeutung 2 werden die beiden frequentesten Verben engager und embaucher als Übersetzungen aufgeführt. Wie oben beim deutsch-englischen Wörterbuch werden auch hier keine Kookkurrenzpartner in der Zielsprache genannt.

Bei Unterbedeutung 3 kommen s'adapter und se préparer vor, die beiden frequentesten Entsprechungen in Europarl, dazu être prêt à $q c$. Darüber hinaus werden drei weitere Übersetzungsvorschläge aufgeführt, die in Europarl nicht auftreten: s'attendre à qc, être organisé en vue de, se régler sur qn. Im selben Abschnitt treten dann noch Äquivalente für unsere Unterbedeutung 5 (gesinnt sein) auf, welche unseres Erachtens besser getrennt zu behandeln wäre.

\footnotetext{
9 In Europarl finden sich insgesamt 38 französische Belege für ein Verfahren einstellen. In den französischen Texten kommen folgende verbale Entsprechungen vor: abandonner: 6 ; classer: 4; annuler: 3 ; mettre fin: 3 ; suspendre: 3 ; interrompre: 2 ; mettre un terme: 2 ; abroger: 1 ; arrêter: 1 ; cesser: 1 ; retirer: 1 ; stopper: 1 ; Diverses: 10. Mit nur einem Beleg kann arrêter auf dieser Grundlage kaum als bevorzugter Kookkurrenzpartner gelten.
} 
Valenzforschung mit Parallelkorpora

2. (anstellen) assumere

6. (aufhören) interrompere, sospendere, cessare: den Betrieb einstellen sospendere l'attività; das

Feuer, die Arbeit einstellen cessare il fuoco, interrompere il lavoro

7. DIR. archiviare: ein Verfahren einstellen archiviare un processo

9. BUROCR. (in Anschlag bringen) calcolare, computare

3. (sich vorbereiten) sich auf etw. (A) einstellen prepararsi a qcs.

4. (sich anpassen) sich auf jdn, etw. einstellen adattarsi a qen., a qcs.

\section{Langenscheidt Deutsch - Italienisch}

Für Unterbedeutung 1 werden drei in Europarl frequente Verben genannt (interrompere, sospendere, und cessare), die prominenten Funktionsverbgefüge porre fine / mettere fine / avere fine werden dagegen nicht aufgeführt. Zum Übersetzungsvorschlag sospendere wird der Kookkurrent attività genannt, was mit der Analyse von Europarl übereinstimmt. Die Kookkurrenzpartner fuoco und lavoro (zu cessare bzw. interrompere) erscheinen in Europarl nicht im Kookkurrenzprofil. Das als juristisch ausgewiesene archiviare un processo scheint im Italienischen bloß eine unter mehreren Ausdrucksweisen zu sein. ${ }^{10}$

Für Unterbedeutung 2 wird mit assumere die in Europarl mit Abstand häufigste Entsprechung genannt. Wie bei den anderen untersuchten Wörterbüchern werden auch hier keine Kookkurrenzpartner in der Zielsprache genannt.

Unsere Unterbedeutung 3 (sich auf etw. vorbereiten) wird in diesem Wörterbuch auf zwei Unterbedeutungen aufgeteilt. Als Entsprechungen werden prepararsi und adattarsi gegeben, das in Europarl frequenteste Übersetzungsäquivalent adeguarsi fehlt hingegen. Kookkurrenten werden im Wörterbuch keine genannt.

Als einziges der untersuchten Wörterbücher registriert Langenscheidts Handwörterbuch Deutsch - Italienisch Unterbedeutung 4 (für etw. vorsehen, budgetieren). Allerdings kommt das vorgeschlagene calcolare in den knapp 180 Belegen in Europarl nur gerade einmal vor, computare hingegen überhaupt nicht.

Der Eintrag einstellen im Larousse-Wörterbuch präsentiert sich folgendermassen:

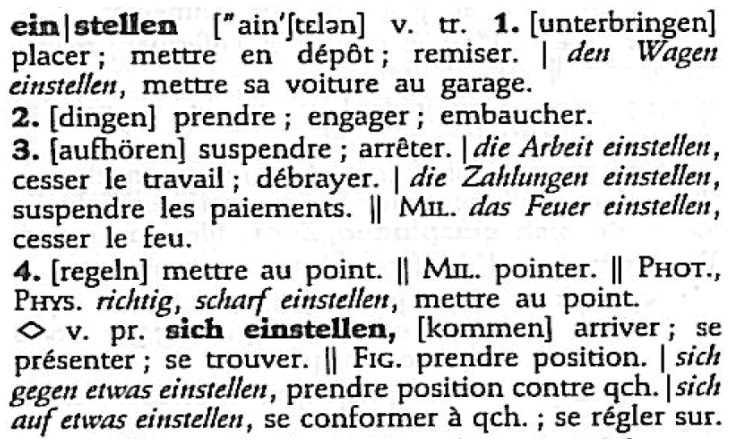

2. [dingen] prendre; engager; embaucher.

3. [aufhören] suspendre; arrêter. |die Arbeit einstellen, cesser le travail ; débrayer. I die Zahlungen einstellen, suspendre les paiements. || Miz. das Feuer einstellen, cesser le feu.

4. [regeln] mettre au point. || MII. pointer. \| Pнот., PHys. richtig, schar einstellen, mettre au point.

$\checkmark$ v. pr. sich einstellen, [kommen] arriver; se présenter; se trouver. II Fig. prendre position. | sich gegen etwas einstellen, prendre position contre qch. |sich auf etwas einstellen, se conformer à qch.; se régler sur.

\section{Larousse (Grappin) Deutsch - Französisch}

Grappin bietet insgesamt vier bzw. fünf Unterbedeutungen. An erster Stelle wird die auch in den Duden-Wörterbüchern jeweils an erster Stelle genannte Unterbedeutung 'unterbringen' (Beispiel: den Wagen einstellen: mettre sa voiture au garage) aufgeführt. Es sei hier noch einmal darauf hingewiesen, dass diese Unterbedeutung in den von uns untersuchten Korpora nicht belegt ist. Sie ist auf jeden Fall höchst marginal und in der Bedeutung 'mettre sa voiture

\footnotetext{
${ }^{10}$ In Europarl finden sich insgesamt 35 italienische Belege für ein Verfahren einstellen. In den entsprechenden Textabschnitten kommen folgende Entsprechungen vor: archiviare: 7; sospendere: 7; ritirare: 4; porre fine, interrompere, abbandonare: je 3; sechs weitere Verben zusammen: 8.
} 
au garage' wohl gar nicht sprachüblich. Die bei der zweiten Unterbedeutung gegebene Semantisierung 'dingen' ist heute veraltet und dürfte deshalb für einen Fremdsprachenlerner kaum hilfreich sein; zudem ist das an erster Stelle vorgeschlagene Übersetzungsäquivalent prendre zu allgemein und darüber hinaus umgangssprachlich. Auch sonst sind die Übersetzungsäquivalente zum Teil unglücklich gewählt. So dürfte débrayer bei der dritten Unterbedeutung in den meisten Fällen nicht passen, weil dieses Verb nur im Zusammenhang mit Streikhandlungen und mit technischen Geräten verwendet wird. Die vierte Unterbedeutung mettre au point ist insofern zu spezifisch, als dieses Syntagma nicht allgemein für das Regeln von Geräten gebraucht werden kann. Die in der Infinitivform angesetzte Verbgruppe sich gegen etwas einstellen ist nicht sprachüblich; effektiv gebraucht wird diese Wendung nämlich nur in Form des Partizips II (gegenüber etw./jdm. positiv usw. eingestellt sein). Alles in allem: Der Wörterbucheintrag einstellen im Larousse-Wörterbuch - zweifellos einem der besten gegenwärtig auf dem Markt befindlichen deutsch-französischen Wörterbücher - weist mehrere Ungenauigkeiten, Verzerrungen und Fehler auf und kann damit nicht den Anspruch erheben, ein wirklichkeitsgetreues Abbild der sprachlichen Fakten zu geben.

Interessant ist auch ein Blick auf die - heute von vielen Benutzern als erstes konsultierten Online-Wörterbücher auf dem Internetportal www.leo.org. Deren Angaben sind jedoch nicht nach Unterbedeutungen gegliedert und wirken entsprechend unübersichtlich. Der hier gebotene ungeordnete Wust von Informationen ist eigentlich nur für jemanden von Nutzen, der die Zielsprache, über die er eigentlich etwas wissen will, bereits gut kennt. Für fortgeschrittene Nutzer von einem gewissen Interesse sind hier jedoch die Diskussionsforen, in denen von - mit den Angaben unzufriedenen - "Leonauten" ganz gezielt nach einzelnen Unterbedeutungen oder Verwendungsweisen gefragt wird, z. B. beim Verb einstellen nach der Übersetzung von Syntagmen wie Skibindung einstellen, Lieferung einstellen, sich auf einen Schüler einstellen, im Internet einstellen, einen Rekord einstellen usw.

\section{Vorschlag für einen Wörterbucheintrag des Verbs einstellen}

Wie anderswo gilt auch hier: Kritisieren ist leicht, selber besser machen schon wesentlich schwieriger. Wenn wir nun im Lichte der empirisch gewonnenen neuen Erkenntnisse zum Verb einstellen einen Eintrag für ein Schülerwörterbuch verfassen wollten, würden wir uns auf Überlegungen verschiedener Natur abstützen. ${ }^{11}$ Die drei frequentesten Unterbedeutungen sowohl in DeReKo als auch in Europarl ('mit etwas aufhören', 'anstellen', 'sich auf etw. vorbereiten') müssen aber unseres Erachtens ihren festen Platz in einem solchen Nachschlagewerk haben, wobei man das reflexive sich einstellen aus praktischen Erwägungen am Ende aufführen könnte. Auf die Aufnahme der vierthäufigsten Unterbedeutung 'budgetieren' kann in einem solchen Nachschlagewerk verzichtet werden, weil diese von der Domäne her zu spezifisch ist. Hingegen scheint uns die Aufnahme der fünfthäufigsten Unterbedeutung 'regeln, justieren' angesichts der Bedeutung von technischen Geräten in der heutigen Welt sehr sinnvoll. Wir würden uns somit in einem Lernerwörterbuch auf die vier folgenden Unterbedeutungen beschränken, deren Semantisierung in diesem Vorschlag durch einige gezielt ausgewählte typische Kookkurrenten unterstützt wird.

\footnotetext{
${ }^{11}$ Der Einsatz von korpuslinguistischen Methoden in der Lexikographie ist auch Gegenstand von Hass (2005).
} 
Valenzforschung mit Parallelkorpora

\section{einlstellen}

1. [mit etw. aufhören] cesser; suspendre; arrêter I die Feindseligkeiten e., cesser les hostilités I die Produktion e., arrêter la production.

2. [anstellen] engager; embaucher; recruter I Personal e., engager/embaucher du personnel.

3. [regeln, justieren] régler; ajuster I ein Gerät e., régler un instrument.

4. sich einstellen auf etw./jdn. [s. auf etw./jdn. vorbereiten] s'adapter à; se préparer à I s. auf eine neue Situation e., s'adapter à une nouvelle situation I $s$. auf einen Wechsel e. müssen, devoir se préparer à un changement.

\section{Schlussbemerkung}

Zusammenfassend kann man im Anschluss an diese Untersuchung festhalten, dass Parallelkorpora für die zweisprachige Valenzforschung und Lexikographie von hohem Interesse sind. Auf der Grundlage einer Handauszählung der Unterbedeutungen eines Lexems in einer Ausgangssprache lassen sich die Entsprechungen in den Vergleichssprachen quantifizieren. So können wir beispielsweise behaupten, dass für ${ }^{1}$ einstellen im Französischen ein gutes Dutzend Übersetzungsmöglichkeiten existieren, von denen eine klar dominiert (cesser) und die drei folgenden etwa gleich häufig auftreten (suspendre, mettre un terme, arrêter). Über diese reinen Größenrelationen hinaus können aber auch verlässliche Aussagen über die typischen Verwendungskontexte und Kookkurrenzpartner gemacht werden. Bei cesser beispielsweise sind die Nomina activités, violence und hostilités prominent, bei arrêter dagegen production.

Zurzeit ist es allerdings immer noch so, dass die verfügbaren Parallelkorpora zu wenig umfangreich und die entsprechenden Recherchewerkzeuge zu wenig elaboriert und fein sind. Das hat zur Folge, dass die oben beschriebenen drei Teilschritte gegenwärtig noch ziemlich aufwändig sind. Es wäre wünschenswert, wenn die Analysetools - zur Vermeidung von lästigen Zwischenschritten - direkt mit den Korpora verknüpft werden könnten. Eines scheint aber schon heute klar: Die zunehmende Automatisierung von Analyseverfahren wird die Handhabbarkeit auch von sehr großen Textmengen möglich machen und damit einen wichtigen Beitrag zu der - im Zeitalter der Globalisierung dringend notwendigen - Verbesserung der einsprachigen und vor allem der zweisprachigen Wörterbücher leisten.

\section{Literatur}

Church, Kenneth W./Mercer, Robert L. (1993): "Introduction to the Special Issue on Computational Linguistics. Using Large Corpora". Computational Linguistics 19/1: 1-24.

Duffner, Rolf/Näf, Anton (2006): "Digitale Textdatenbanken im Vergleich". Linguistik online 28: 7-23. www.linguistik-online.org/28_06/duffnerNaef.html (Stand: September 2009).

Hass, Ulrike (ed.) (2005). Grundfragen der elektronischen Lexikographie. elexiko - das Online-Informationssystem zum deutschen Wortschatz. Tübingen.

Institut für Deutsche Sprache (ed.): COSMAS II. Corpus Search, Management and Analysis System. www.ids-mannheim.de/cosmas2/ (Stand: September 2009).

Institut für Deutsche Sprache (ed.) (1999-2002): DeReKo I. Deutsches Referenzkorpus. www.ids-mannheim.de/kl/projekte/korpora/ (konsultiert: August/September 2008).

Kamber, Alain (2008): Funktionsverbgefüge - empirisch. Eine korpusbasierte Untersuchung zu den nominalen Prädikaten des Deutschen. Tübingen.

Langenscheidt-Redaktion (ed.) (2006): Langenscheidt e-Handwörterbuch Deutsch - Englisch 5.0. Wörterbuch auf CD-ROM. Berlin/München. 
Langenscheidt-Redaktion (ed.) (2007): Langenscheidt e-Handwörterbuch Deutsch - Französisch 5.0. Wörterbuch auf CD-ROM. Berlin/München.

Langenscheidt-Redaktion (ed.) (2003): Langenscheidt e-Handwörterbuch Deutsch - Italienisch 4.0. Wörterbuch auf CD-ROM. Berlin/München.

Lemnitzer, Lothar/Zinsmeister, Heike (2006): Korpuslinguistik. Eine Einführung. Tübingen.

Pierre Grappin et al. (eds.) (1999): Larousse Wörterbuch Französisch - Deutsch; Deutsch Französisch. Paris.

Scherer, Carmen (2006): Korpuslinguistik. Heidelberg.

Tiedemann, Jörg: OPUS. An Open Source Parallel Corpus. http://urd.let.rug.nl/tiedeman/ OPUS/index.php (konsultiert zwischen September 2008 und Februar 2009). 